\title{
Preparation of Fluorosilicone Triblock Copolymers and Microphase Separation Behavior on Surfaces
}

\author{
Xitao Cheng ${ }^{1}$,, Xuan Tang ${ }^{2}$, Wenhong $\mathrm{Li}^{3}$, Fangfang Huang ${ }^{1}$, Qianjin Wang ${ }^{1}$ \\ ${ }^{1}$ Shaanxi Provincial Research and Design Institute of Petroleum and Chemical Industry, Xi'an, China \\ ${ }^{2}$ College of Chemistry and Chemical Engineering, Xi'an Shiyou University, Xi'an, China \\ ${ }^{3}$ School of Chemical Engineering, Northwest University, Xi'an, China
}

Email address:

chengxitao@126.com (Xitao Cheng), tangxuan@xsyu.edu.cn (Xuan Tang)

${ }^{*}$ Corresponding author

\section{To cite this article:}

Xitao Cheng, Xuan Tang, Wenhong Li, Fangfang Huang, Qianjin Wang. Preparation of Fluorosilicone Triblock Copolymers and Microphase Separation Behavior on Surfaces. American Journal of Polymer Science and Technology. Vol. 5, No. 4, 2019, pp. 105-113. doi: 10.11648/j.ajpst.20190504.12

Received: October 25, 2019; Accepted: November 18, 2019; Published: November 26, 2019

\begin{abstract}
Fluorosilicone polymer is a new type of structure of the fluorine silicon block polymer. Because it combines the excellent properties of organic silicone and organic fluorine compounds, it has a wide range of uses and becomes a hot issue in the field of materials. In the paper, A series of novel poly(2,2,3,4,4,4-hexafluorobutyl methacrylate)-blockpoly(dimethylsiloxane)-block-poly(2,2,3,4,4,4-hexafluorobutyl methacrylate)s (PHFBMA-b-PDMS-b-PHFBMA) were synthesized by atom transfer radical polymerization (ATRP) with different molecular weight prepared polydimethylsiloxane macroinitiator as raw materials. The effects of the fluorine and silicone content on the hydrophobic and oleophobic properties of prepared triblock copolymers were also investigated. The structure and composition of the copolymers were analyzed and identified by infrared spectroscopy (IR) and nuclear magnetic resonance (NMR). The average molecular weight and molecular weight distribution of the prepared PHFBMA-b-PDMS-b-PHFBMA were evaluated by gel permeation chromatography (GPC). The surface energy of the triblock copolymers was calculated from the contact angle reaches as low as $10.43 \mathrm{mN} / \mathrm{m}$ through the Owens-Wendt-Rabel-Kaelble method, with the fluorine content of triblock polymer was $19.0 \mathrm{wt} \%$. Atomic force microscopy (AFM), differential scanning calorimetry (DSC) and X-ray photoelectron spectroscopy (XPS) indicated that there were Obvious nanoscopically microphase separation on the surface of the prepared triblock copolymers and the fluoride contents in the block polymer of the fluorine silicon block were more likely to migrate to the surface.
\end{abstract}

Keywords: Microphase Separation, PHFBMA-b-PDMS-b-PHFBMA, ATRP, Surface Free Energy, Synthesis and Characterization

\section{Introduction}

The fluorinated acrylate/polydimethyl siloxane block copolymer (PDMS-b-PF) is a new type of structure of the fluorine silicon block polymer. Because the main chain contains both soft polysiloxane chains and low surface energy fluorine segments (PF), it has a wide range of uses. The surface of PDMS-b-PF can appear microphase separation when it forms on the substrate surface for the differences in thermodynamics between PDMS and PF [1-3]. And PDMS-b-PF is obtained a very low surface tension [4], shows strong hydrophobic/oilphobic and self-cleaning properties $[5,6]$. The microphase separation is an important feature of the fluorosilicone block copolymers which is one of the hot topics in the field of organic fluorine and silicon materials in recent years [7-9], and itmakes the function of the polymer greatly improved and the application domain widened $[10,11]$. For example, Huang L. B et al [12] added a certain amount of styrene and 1,3,5-tris $\left(1,3,5-\left(3^{\prime}, 3^{\prime}, 3^{\prime}-\right.\right.$ trifluoropropyl) ring siloxane block copolymers to the polystyrene and the surface properties of the blends were studied. The result showed that the surface tension could be reduced, the hydrophobicity and oilphobicity could be improved effectively even if a small amount of block polymer was added to polystyrene. Uyanik and coworkers 
[13] found that the contact angle increased with the polysiloxane segment content orthe molecular weight increased through evaluation of fluorinated silicone block polymer's surface properties. But there is no report on the synthesis, characterization and microphase separation of fluorosilicone triblock copolymers by using 2,2,3,4,4,4hexafluorobutyl methacrylate (HFBMA) as raw material.

In this paper, series of different molecular weight, different fluorine silicon content of PHFBMA-b-PDMS-b-PHFBMA was prepared by atom transfer radical polymerization (ATRP) with using the Cl-PDMS-Cl as the initiator which was synthesized by the reaction of bishydroxypropyl terminated polysiloxane (BHTPS) and $\alpha$-chloroisobutyryl chloride and HFBMA as the monomer. The surface tension and the microphase separation of the membrane surface of the polymers were investigated.

\section{Experimental}

\subsection{Materials}

A series of BHTPS with different hydroxyl content were produced by Shaanxi Provincial Research and Design Institute of Petroleum and Chemical Industry. $\alpha$ chloroisobutyryl chloride (98\%) was obtained from SynAsst Chemical. 2,2,3,4,4,4-hexafluorobutyl methacrylate (HFBMA, 98\%) purchased from Lancaster was washed with $5 \%$ aqueous $\mathrm{NaOH}$ solution to remove the inhibitor. Cuprous chloride obtained from Tianjin Chemical Reagent Factory was washed with glacial acetic acid and methanol. All other materials were analytical reagent and used without further purification.

\subsection{Synthesis of Cl-PDMS-Cl Macroinitiator}

The reaction equation is shown in Figure 1.

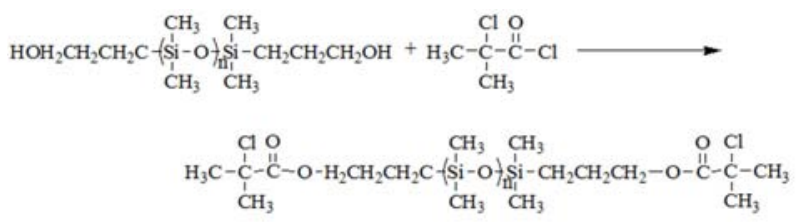

Figure 1. Synthetic scheme for the preparation of Cl-PDMS-Cl.

The certain amount of BHTPS with different hydroxyl content, triethylamine, and $50 \mathrm{~mL}$ tetrahydrofuran were added into four-neck round bottom flask in low temperature reactor, which was equipped with condenser, thermometer, constant pressure funnel and nitrogen conduit. The system was strictly deoxygenated by nitrogen filling time and again, and then cooled to $0^{\circ} \mathrm{C}$, held the temperature and dropped the certain amount of $\alpha$-chloroisobutyryl chloride(BHTPS: $\alpha$ chloroisobutyryl chloride:triethylamine $=1: 2.6: 3.6)$ completely in $30 \mathrm{~min}$. Then warmed up to $25^{\circ} \mathrm{C}$, held the temperature and mixed for $12 \mathrm{~h}$. Insoluble substances were removed by filtration and the mixture was dissolved in $100 \mathrm{~mL}$ dichloromethane after evaporation of tetrahydrofuran. The solution was washed two times with $100 \mathrm{~mL}$ saturated sodium bicarbonate. The organic phase was dried with anhydrous sodium sulfate. The macroinitiator with different molecular weight were obtained by evaporating solvent after filtering.

\subsection{Synthesis of PHFBMA-b-PDMS-b-PHFBMA Triblock Copolymers}

The reaction equation is shown in Figure 2.

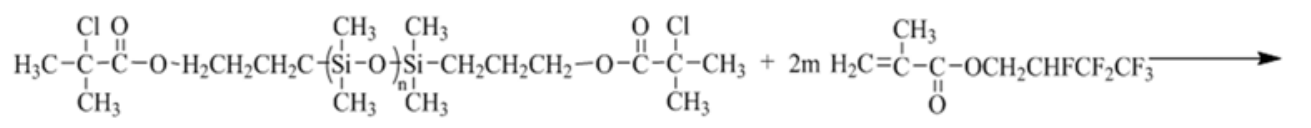

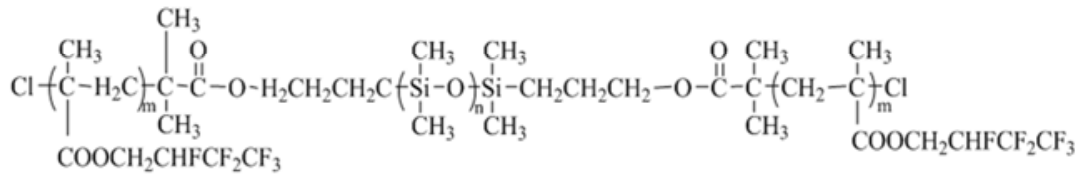

Figure 2. Synthetic scheme for the preparation of PHFBMA-b-PDMS-b-PHFBMA triblock copolymers.

The certain amount of Cl-PDMS-Cl, $\mathrm{HFBMA}, \mathrm{Cu}^{+}$ catalyst, Catalyst ligands([HFBMA]:[Cl-PDMS$\mathrm{Cl}]:[\mathrm{PMPA}+\mathrm{BPY}]:[\mathrm{CuCl}]=20: 1: 2: 1)$ and $50 \mathrm{~mL}$ toluene were added into four-neck round-bottom flask, which was equipped with condenser, thermometer, constant pressure funnel and nitrogen conduit. The system was strictly deoxygenated by nitrogen filling time and again, and then warmed up to the specified temperature, held the temperature for reacting in a certain time. The mixture was diluted with $50 \mathrm{~mL}$ tetrahydrofuran, through neutral alumina column to remove the catalyst, the treated liquid was precipitated in 50 $\mathrm{mL}$ methanol. The obtained polymer was rinsed with methanol for three times and dried to constant weight in vacuum drying box at $40^{\circ} \mathrm{C}$.

\subsection{Characterizations}

The product was analyzed by VECTOR-22 infraredspectrometer with resolution of $4.0 \mathrm{~cm}^{-1}$ and the average data of ten times test. ${ }^{1} \mathrm{HNMR}$ and CNMR spectra were measured on a Varian INOVA-400 NMR spectrometer with deuterated chloroform as the solvent. GPC was performed by using THF as the eluent at a flow rate of $1 \mathrm{~mL} / \mathrm{min}$ under $25^{\circ} \mathrm{C}$, with a Waters 1525 Binary HPLC pump equipped with a Waters 2414 refractive index detectorandWaters HT 2,3,4 columns and monodisperse polystyrene standards were used for calibration. Glass transition temperature was determined by Star e Series 
DSC1 under the protection of nitrogen with heating rate of $10 \mathrm{~K} / \mathrm{min}$. Static water/oil contact angle of the triblock copolymer film was measured on a telescopic goniometer (JC2000D3A). For each angle reported, five sample readings from different surface locations were averaged. The AFM observation was made on Innova SPM (Veeco Instruments, America) in ultra-light tapping mode at room temperature equipped with MLCT-MT-A tip, using the microfabrication cantilevers with a spring constant of approximately $0.05 \mathrm{Nm}^{-1}$. All AFM data including the height, phase shift and the three dimensional (3D) image as well were recorded simultaneously. X-ray photoelectron spectroscopy (XPS) measurement was conducted using a $\mathrm{K}_{\alpha}$ spectrometer (Axis Ultra, Kratos Analytical Ltd., U.K.), and the core level spectra were measured using a monochromatic $\mathrm{Al} \mathrm{K}_{\alpha} \mathrm{X}$-ray source $(\mathrm{hv}=1486.7 \mathrm{eV})$. The analyzer was operated at $23.5 \mathrm{eV}$ pass energy, and the analyzed area was $200-800 \mu \mathrm{m}$ in diameter. The lowest energy resolution was $0.48 \mathrm{eV}(\mathrm{Ag} 3 \mathrm{~d} 5 / 2)$. Binding energies were referenced to the adventitious hydrocarbon $\mathrm{C}$ 1s line at $285.0 \mathrm{eV}$, and the curve fitting of the XPS spectra was performed using the least-squares method. The analyzer chamber pressure was $1.06 \times 10^{-8} \mathrm{~Pa}$. Narrow scan spectrometer of C $1 \mathrm{~s}, \mathrm{O} 1 \mathrm{~s}, \mathrm{~N} \mathrm{~s}$ and Si $2 p$ were collected and peak analysis were carried out by PHIMATLAB software.

\section{Results and Discussion}

\subsection{Characterization of PHFBMA-b-PDMS-b-PHFBMA and $\mathrm{Cl}-\mathrm{PD} \mathrm{MS}-\mathrm{Cl}$}

The IR of Cl-PDMS-Cl and PHFBMA-b-PDMS-bPHFBMA are shown in Figure 3.

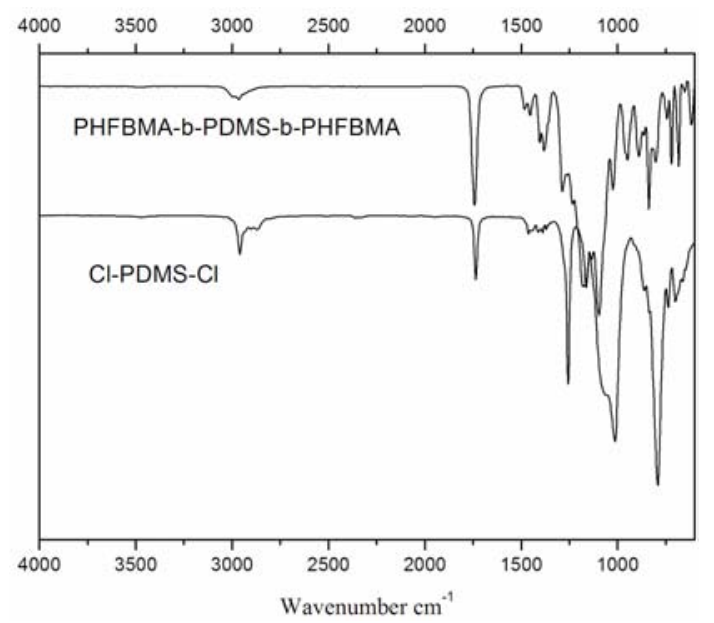

Figure 3. IR of Cl-PDMS-Cl and PHFBMA-b-PDMS-b-PHFBMA.

Figure 3 showed that from Cl-PDMS-Cl to PHFBMA-bPDMS-b-PHFBMA at $1740 \mathrm{~cm}^{-1}$ there was the stretching vibration of carbonyl group developed from nothing to tender to stronger and no peak appear at $1640 \mathrm{~cm}^{-1}$ which belonged to flex oscillatory absorption of $\mathrm{C}=\mathrm{C}$ group. At the same time, three peaks centered at $1283 \mathrm{~cm}^{-1}, 1184 \mathrm{~cm}^{-1}, 915 \mathrm{~cm}^{-1}$, corresponding to the antisymmetric and symmetric stretching vibrations of the $-\mathrm{CF}_{3}$ group, two peaks centered at $723 \mathrm{~cm}^{-1}$,
$564 \mathrm{~cm}^{-1}$ belonged to a combination of the cocking and wagging vibrations of $-\mathrm{CF}_{2}$ - group, and the characteristic peaks of the PDMS block appeared between $1060 \mathrm{~cm}^{-1}$ and $1260 \mathrm{~cm}^{-1}$ still existed. All of these indicated that PHFBMAb-PDMS-b-PHFBMA was synthesized completely.

The raw material and product were charactered by NMR, Figure $4 \mathrm{a}$ and Figure $4 \mathrm{~b}$ were the ${ }^{1} \mathrm{HNMR}$ of Cl-PDMS-Cl and PHFBMA-b-PDMS-b-PHFBMA, Figure $4 c$ was the ${ }^{13}$ CNMR of PHFBMA-b-PDMS-b-PHFBMA.
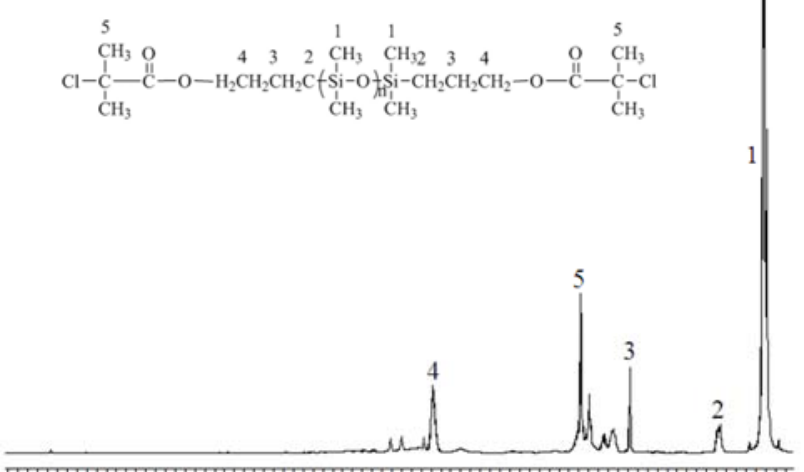

$\begin{array}{llllllllllllllllll}7.5 & 7.0 & 6.5 & 6.0 & 5.5 & 5.0 & 4.5 & 4.0 & 3.5 & 3.0 & 2.5 & 2.0 & 1.5 & 1.0 & 0.5 & 0.0\end{array}$

(a) ${ }^{1} \mathrm{HNMR}$ of Cl-PDMS-Cl

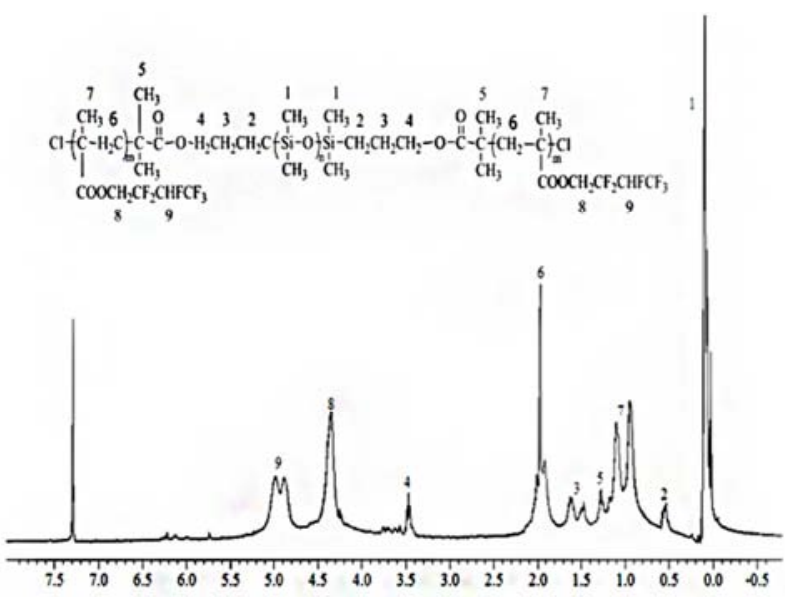

(b) ${ }^{1}$ HNMR of PHFBMA-b-PDMS-b-PHFBMA

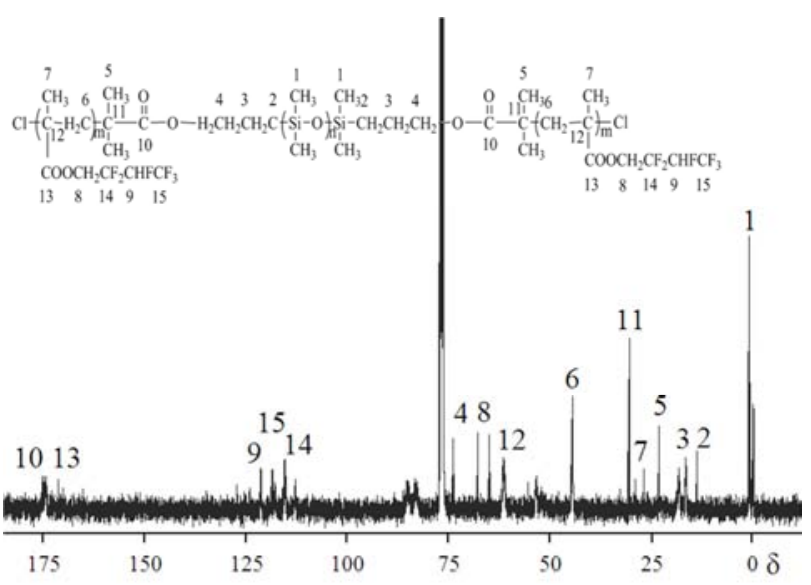

(c) ${ }^{13} \mathrm{CNMR}$ of PHFBMA-b-PDMS-b-PHFBMA

Figure 4. NMR characterization. 
In Figure 4b, the chemical shift at $4.95 \mathrm{ppm}$ belonged to CHF-, the other hydrogen atom chemical shift assignments were shown in Figure $4 \mathrm{~b}$ tagging. It also could be seen that the characteristic peaks of double bond hydrogen of $\mathrm{CH}_{2}=\mathrm{CCHCO}$ didn't appear. Likewise, CNMR in Figure 4c there was no peak at $6.14 \mathrm{ppm}$ which was attributed to the double bond carbon of $\mathrm{CH}_{2}=\mathrm{CCHCO}$, the chemical shifts at $116.54 \mathrm{ppm}, 121.98 \mathrm{ppm}, 119.45 \mathrm{ppm}$ were caused by $-\mathrm{CF}_{2^{-}}$, $\mathrm{CHF}-,-\mathrm{CF}_{3}$ - respectively and the other carbon atom chemical shift assignments were shown in Figure 4c tagging.

A series of PHFBMA-b-PDMS-b-PHFBMA with different fluorine content were synthesized by ATRP with different molecular weight Cl-PDMS-Cl and HFBMA as raw materials. The average molecular weight and molecular weight distribution of the prepared PHFBMA-b-PDMS-bPHFBMA were evaluated by GPC, the results were shown in Table 1.

Table 1. The average molecular weight and molecular weight distribution of the prepared fluorosilicone triblock copolymers.

\begin{tabular}{lllll}
\hline molecular weight of Cl-PDMS-Cl & Mn (GPC) & Mn (TH) & Mw/Mn & Rate/\% \\
\hline 1950 & 7082 & 7156 & 1.12 & 90.5 \\
2450 & 7582 & 7685 & 1.15 & 91.0 \\
3635 & 8367 & 8824 & 1.14 & 90.2 \\
5030 & 9586 & 10231 & 1.14 & 90.4 \\
6901 & 10560 & 11780 & 1.15 & 90.8 \\
11550 & 16557 & 18762 & 1.22 & 94.5 \\
\hline
\end{tabular}

It could be seen from Table 1 that the conversion rates of the polymerization reaction and the yields of the products were higher, meanwhile the measured molecular weights were also in good agreement with the designed molecular weights. The above analysis showed that macromolecular initiator was reacted with HFBMA thoroughly and the desired product was obtained.

\subsection{Performance and Surface Free Energies}

The PHFBMA-b-PDMS-b-PHFBMA triblock copolymers were expected to be materials with low surface energies. The triblock copolymer surface properties were also investigated through static contact angle measurement, the results of water and oil static contact angel were shown in Figure 5, the effect of DMS and HFBMA block length on static contact angles were shown in Figure 6.

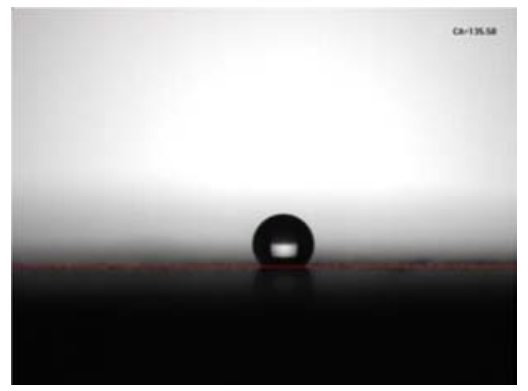

(a) Water static contact angel

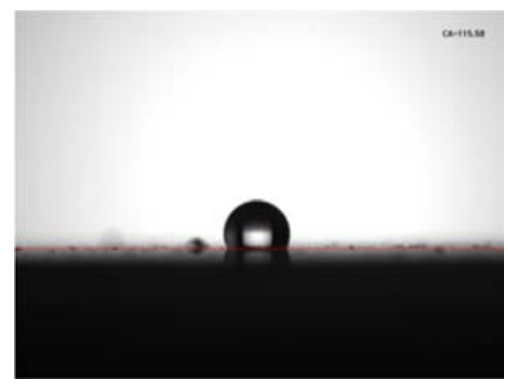

(b) Oil static contact angel

Figure 5. Triblock copolymer surface properties.

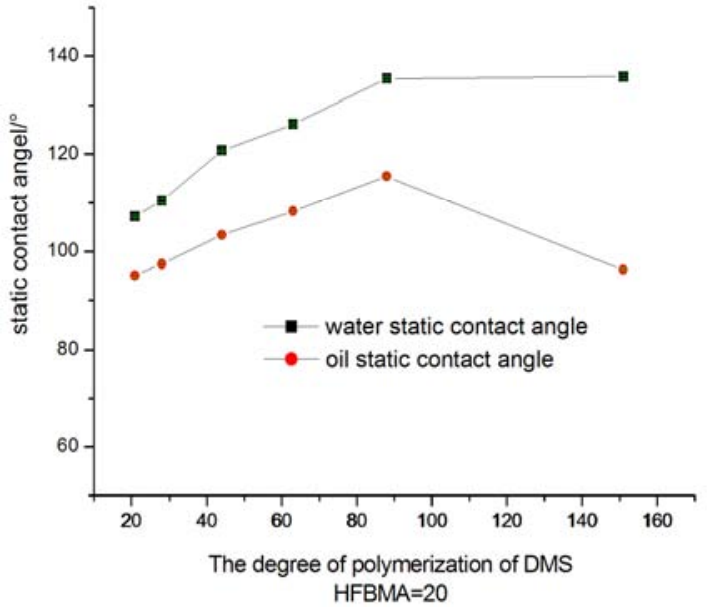

(a) Effects of DMSblock length

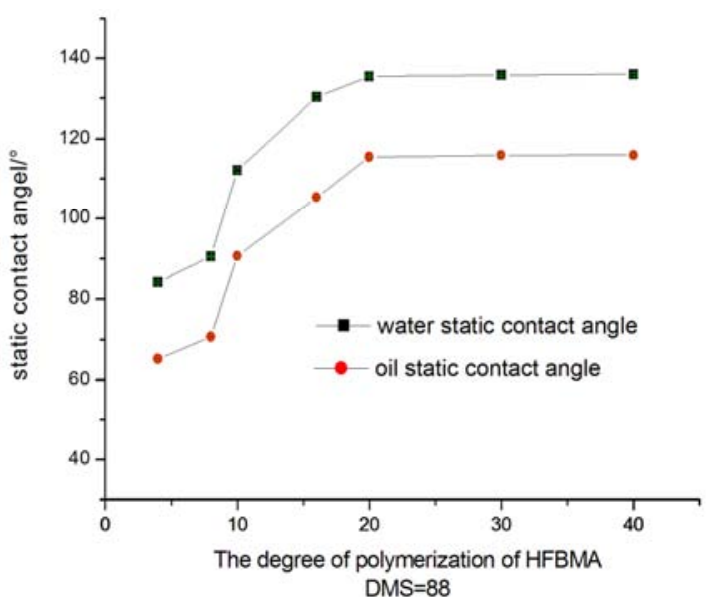

(b) Effects of HFBMA block length

Figure 6. Thestatic contact angel of different degree of polymerization of $D M S$ and HFBMA.

It could be seen from Figure 6 that the water and oil static contact angles toward the air-side surface of the copolymer films increased gradually with DMS block length increased. But when the DMS block length was above 88 , the water and 
oil static contact angles decreased. Similarly the water and oil static contact angles rose with HFBMA block length prolonged. But when the HFBMA block length reached to more than 20 , the growth rate of water and oil static contact angles changed little. The maximum standard deviation in Figure 4 was only $0.50^{\circ}$, which demonstrated that the test experiments had a good reproducibility.

It was mean that the water and oil static contact angles have no monotonic increase or decrease as the DMS or HFBMA increased. It was well known that the fluorinated chain segments of fluorinated silicone block copolymer were easy to be enriched on the surface of copolymer to form the microphase separation because of its low surface energy. The microphase separation of polymer surface was not obvious when the fluorinated chain segments were less, and the performance of hydrophobic and oilphobic was poor. The increase of the fluorinated chain segments were beneficial to the migration which led to the obvious microphase separation. Thus the water and oil static contact angles increased. When the degree of polymerization of DMS was 88 and the degree of polymerization of HFBMA was 20, the fluorinated chain segments on the membrane surface spread and just wrapped the silicon chain. The spreading of fluorinated chain segments could not be increased remarkably and the growth rate of water and oil static contact angles changed little as the fluorinated chain segments increased continuously [14].

And now the surface energy of the PHFBMA-b-PDMS-bPHFBMA triblock copolymer films could be as low as $10.43 \mathrm{mN} / \mathrm{m}$, corresponding to a fluorine content as high as $19.0 \mathrm{wt} \%$. Surface energies of the copolymers were calculated from water and glycerol contact angles. The following Owens-Wendt-Rabel-Kaelble method (OWRK) [15-17] which was derived by Owens and Fang was applied to calculate the surface energies:

$$
\sigma_{l}(1+\cos \theta)=2\left(\sqrt{\sigma_{s}^{d} \sigma_{l}^{d}}+\sqrt{\sigma_{s}^{p} \sigma_{l}^{p}}\right)
$$

If the contact angles of two different liquids on the same polymer surface were known, $\sigma_{\mathrm{s}}{ }^{\mathrm{d}}$ and $\sigma_{\mathrm{s}}^{\mathrm{p}}$ could be obtained from equation 1 . In this study, deionized water and glycerol were selected as the probe liquid to determine the surface free energies of copolymer films. The values of $\sigma_{1}{ }^{\mathrm{d}}(21.80 \mathrm{mN}$ $\left.\mathrm{m}^{-1}\right), \sigma_{\mathrm{l}}^{\mathrm{p}}\left(51.00 \mathrm{mN} \mathrm{m}^{-1}\right)$ and $\theta\left(135.50^{\circ}\right)$ for water and $\sigma_{1}{ }^{\mathrm{d}}$ $\left(34 \mathrm{mN} \mathrm{m}^{-1}\right), \sigma_{\mathrm{l}}^{\mathrm{p}^{\prime}}\left(30 \mathrm{mN} \mathrm{m}^{-1}\right)$ and $\theta^{\prime}\left(115.50^{\circ}\right)$ for glycerol were used in the calculation [17-19]. Thus the surface free energy of the polymer film could be calculated by the equation 2

$$
\begin{gathered}
\sigma_{s}=\sigma_{s}^{d}+\sigma_{s}^{p}=\frac{\left[\left(\sigma_{l}^{d^{\prime}}+\sigma_{l}^{p^{\prime}}\right) \sqrt{\sigma_{l}^{p}}(1+\cos \theta)-\left(\sigma_{l}^{d}+\sigma_{l}^{p}\right) \sqrt{\sigma_{l}^{p^{\prime}}}(1+\cos \theta)\right]^{2}}{4\left(\sqrt{\sigma_{l}^{p} \sigma_{l}^{d^{\prime}}}-\sqrt{\sigma_{l}^{d} \sigma_{l}^{p^{\prime}}}\right)^{2}} \\
+\frac{\left[\left(\sigma_{l}^{d}+\sigma_{l}^{p}\right) \sqrt{\sigma_{l}^{d^{\prime}}}(1+\cos \theta)-\left(\sigma_{l}^{d^{\prime}}+\sigma_{l}^{p^{\prime}}\right) \sqrt{\sigma_{l}^{d}}(1+\cos \theta)\right]^{2}}{4\left(\sqrt{\sigma_{l}^{p} \sigma_{l}^{d^{\prime}}}-\sqrt{\sigma_{l}^{d} \sigma_{l}^{p^{\prime}}}\right)^{2}}
\end{gathered}
$$

Compared to the PDMS and PHFBMA, the surface energy of the triblock copolymers were much lower. Obviously, such low surface free energy should be related to the surface microphase separation of the triblock copolymer. In order to reveal the microphase separation behavior of PHFBMA-bPDMS-b-PHFBMA, the following research activities had been performed.

\subsection{Microphase Separation Behavior of the Surface of PHFBMA-b-PDMS-b-PHFBMA}

Because there were differences between polysiloxane chains and fluorinated segments on the surface tension and the solubility parameter of the prepared triblock copolymers, it could not only form phase separation but also maintain the PDMS and PF unique physical and chemical properties. Thus there would be a number of glass transition temperatures [20]. Figure 7 was the DSC curve of prepared copolymers.

DSC analysis in Figure 7 displayed that two sharp glass transitions, one at $-123^{\circ} \mathrm{C}$ and another at $52^{\circ} \mathrm{C}$ corresponding to the glass transition temperatures $\left(\mathrm{T}_{\mathrm{g}} \mathrm{s}\right)$ of the siloxane segments and fluoride segments respectively, further confirmed that there was a very clear microphase separation in the triblock copolymers.

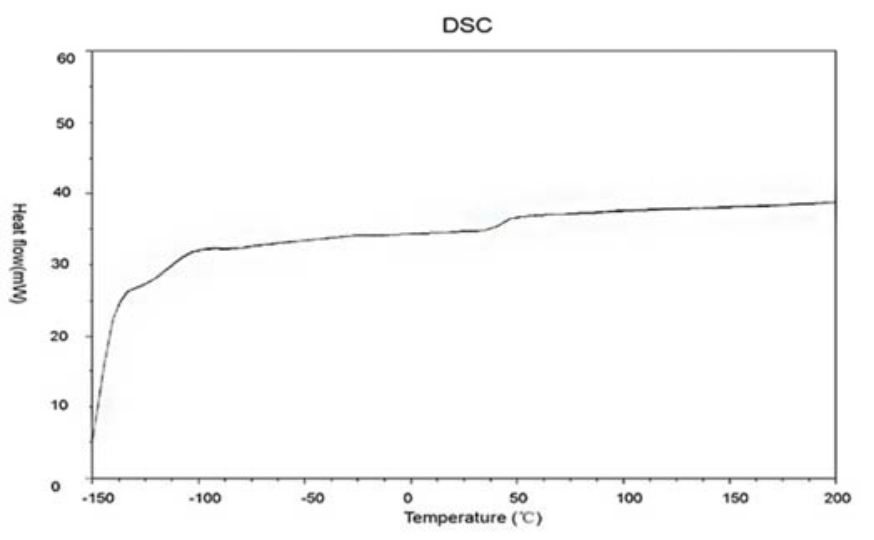

Figure 7. DSC of PHFBMA-b-PDMS-b-PHFBMA.

The surface microscopic morphology of the copolymers film determined the infiltration of water and oil on the surface of the copolymers. It was important to study the surface microscopic morphology of block copolymers. In this study, atomic force microscope(AFM) was used to characterize the microstructure of the prepared copolymers with different chain segments. Figure 8 illustrated the AFM images of the prepared triblock copolymers coatings with different fluorine content. Among them, $\mathrm{a}, \mathrm{b}$ and $\mathrm{g}$ were height, topographic mode and phase mode of $\mathrm{HFBMA}_{5} \mathrm{DMS}_{88} \mathrm{HFBMA}_{5}, \mathrm{c}, \mathrm{d}$ and $\mathrm{h}$ were height, topographic mode and phase mode of $\mathrm{HFBMA}_{10} \mathrm{DMS}_{88} \mathrm{HFBMA}_{10}$, e, f and $\mathrm{i}$ were height, topographic mode and phase mode of $\mathrm{HFBMA}_{12} \mathrm{DMS}_{88} \mathrm{HFBMA}_{12}$. It could be seen from height and topographic images that the surface of the polymer film presented different microscopic morphology and the shape of the surface was irregular for the self assembly of block polymers on the surface. It also could be found that the needle shape of the sample was clustered on the surface which was proved to be to be fluorinated component $[19,20]$, and the nadel became more obvious with the fluoride content increased. The height variation and rough topography exhibited across 
surface were believed to be the result of the phase separation. The phase images showed that there were different degrees of microphase separation in the surface of the block polymers with different ratios of fluoride and silicon. At the same time, the more content of fluoride, the rougher of the film surface, the bigger of white nadel area and the clearer of the boundary between different components, and the morphology of the copolymers were more regular and orderly, the phase separation was more obvious. The Figure 6 revealed that the surface of the film was completely occupied by fluorine content when the PHFBMA chain was 20 , the difference were very small of topographic image and phase image when the PHFBMA chain was 20 and 24. The roughness of $\mathrm{HFBMA}_{5} \mathrm{DMS}_{88} \mathrm{HFBMA}_{5}$ was 1.41 , the $\mathrm{HFBMA}_{10} \mathrm{DMS}_{88} \mathrm{HFBMA}_{10}$ was 3.56 and $\mathrm{HFBMA}_{12} \mathrm{DMS}_{88} \mathrm{HFBMA}_{12}$ was 2.98 which were calculated by nanascope analysis1.4, simultaneously the water/oil static contact angles were $112.16^{\circ} / 90.74^{\circ}, 135.5^{\circ} / 115.5^{\circ}$ and $131.28^{\circ} / 109.83^{\circ}$ respectively. It could be seen from above that the roughness of the triblock copolymers surface affected contact angles greatly.

There was a large variation between PDMS and PHFBMA on the thermodynamic compatibility. It usually tended to be concentrated on the surface of the membrane and the formation scale of the film surface was significantly larger than that of the bulk. In order to make a quantitative analysis of the chemical composition on the surface of the prepared copolymers film, further investigations were underway.

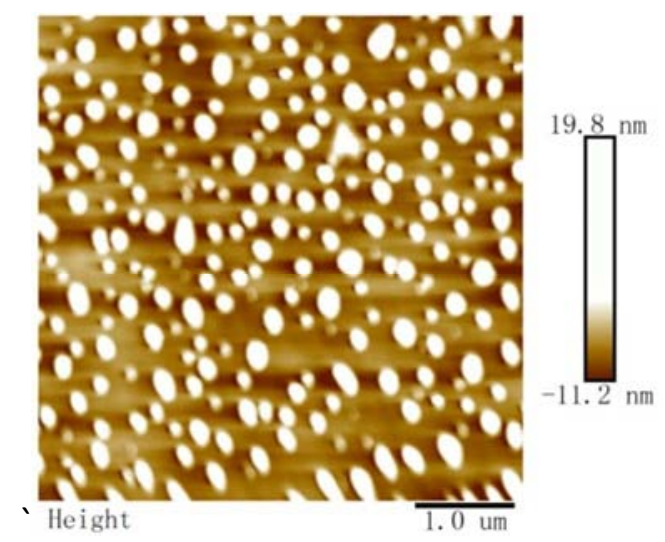

(a) Height of $\mathrm{HFBMA}_{5} \mathrm{DMS}_{88} \mathrm{HFBMA}_{5}$

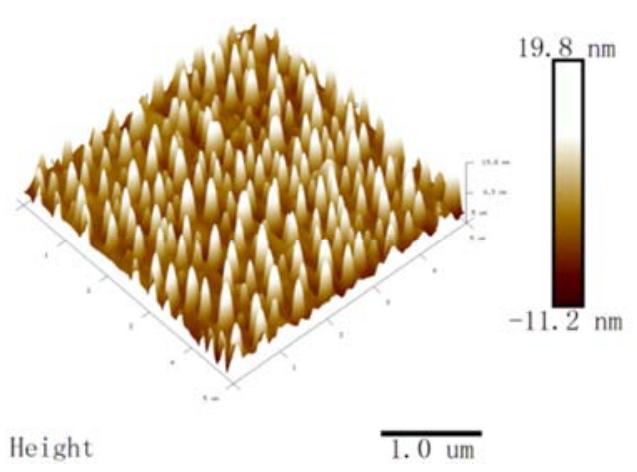

(b) Topographic mode of HFBMA DMS $_{88} \mathrm{HFBMA}_{5}$

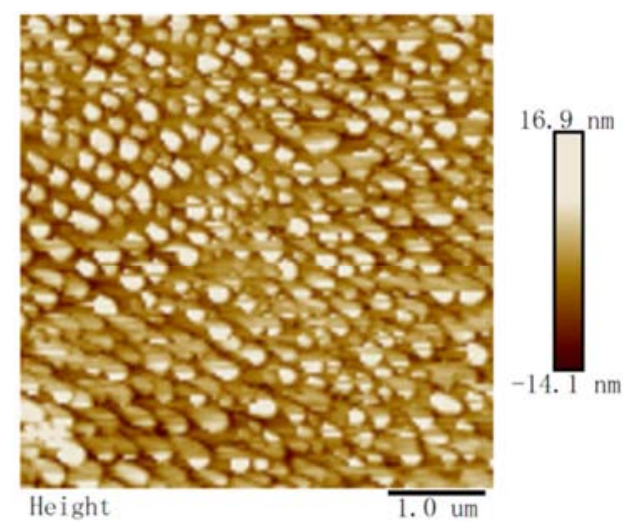

(c) Height of $\mathrm{HFBMA}_{10} \mathrm{DMS}_{88} \mathrm{HFBMA}_{10}$

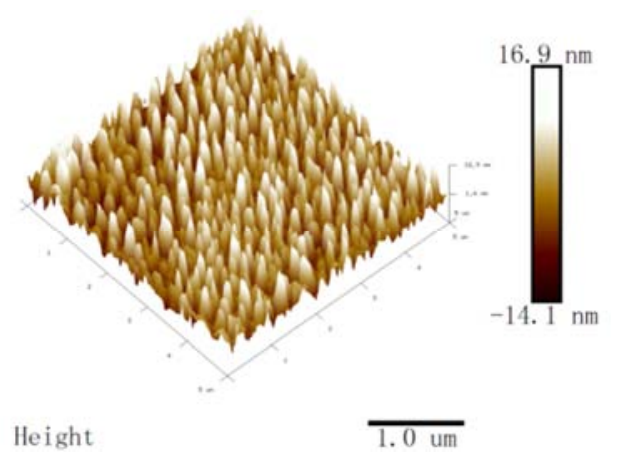

(d) Topographic mode of $\mathrm{HFBMA}_{10} \mathrm{DMS}_{88} \mathrm{HFBMA}_{10}$

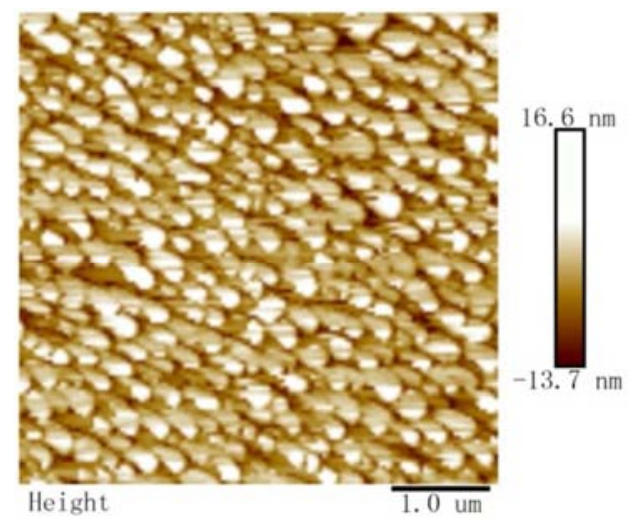

(e) Height of $\mathrm{HFBMA}_{12} \mathrm{DMS}_{88} \mathrm{HFBMA}_{12}$

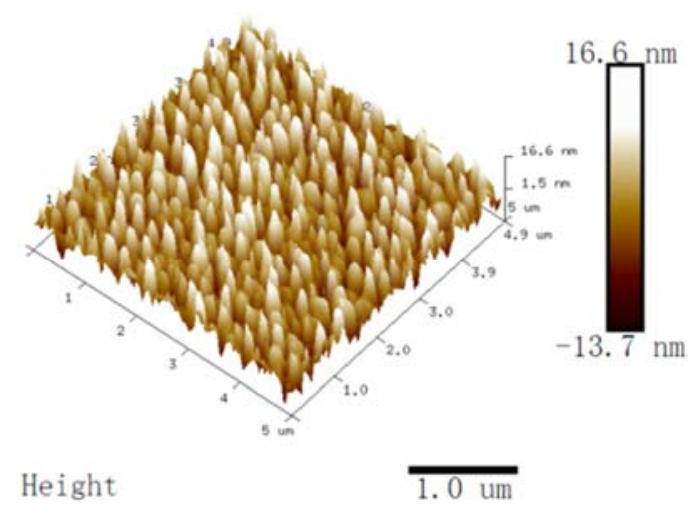

(f) Topographic mode of $\mathrm{HFBMA}_{12} \mathrm{DMS}_{88} \mathrm{HFBMA}_{12}$ 


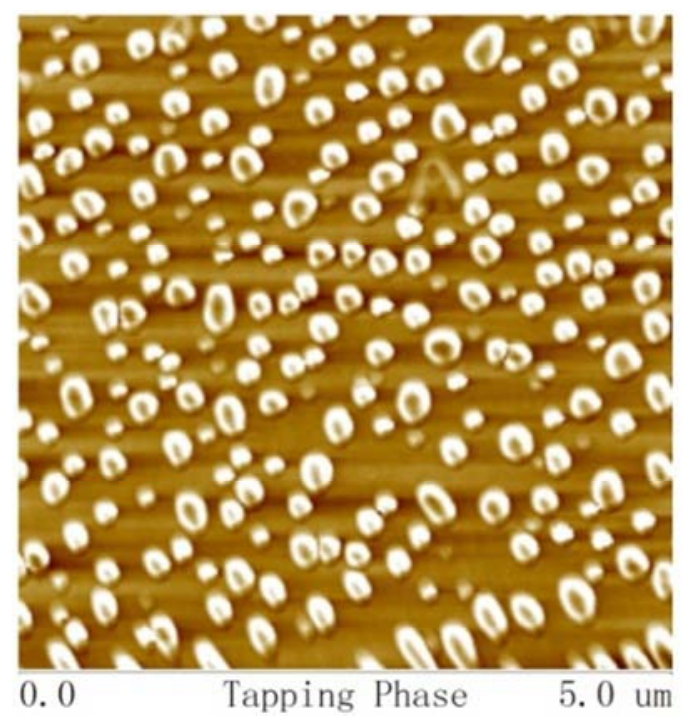

(g) Phase mode of $\mathrm{HFBMA}_{5} \mathrm{DMS}_{88} \mathrm{HFBMA}_{5}$

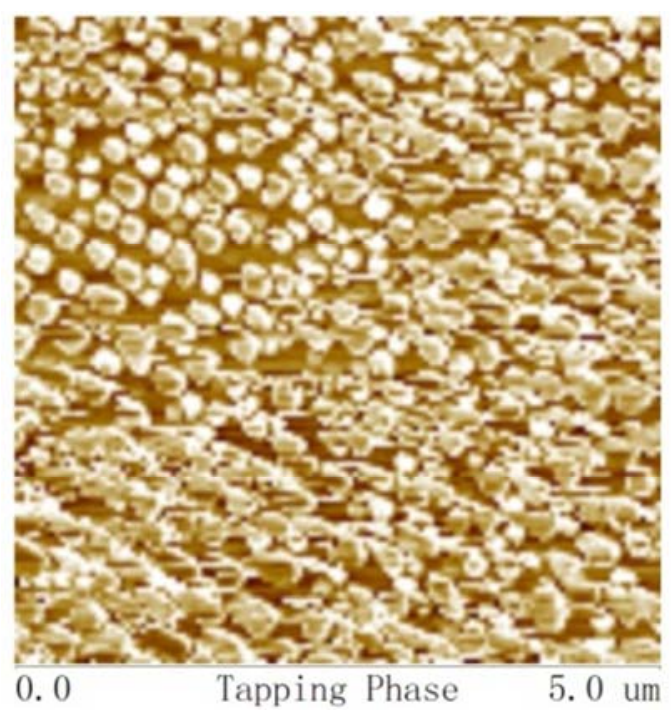

(h) Phase mode of $\mathrm{HFBMA}_{10} \mathrm{DMS}_{88} \mathrm{HFBMA}_{10}$
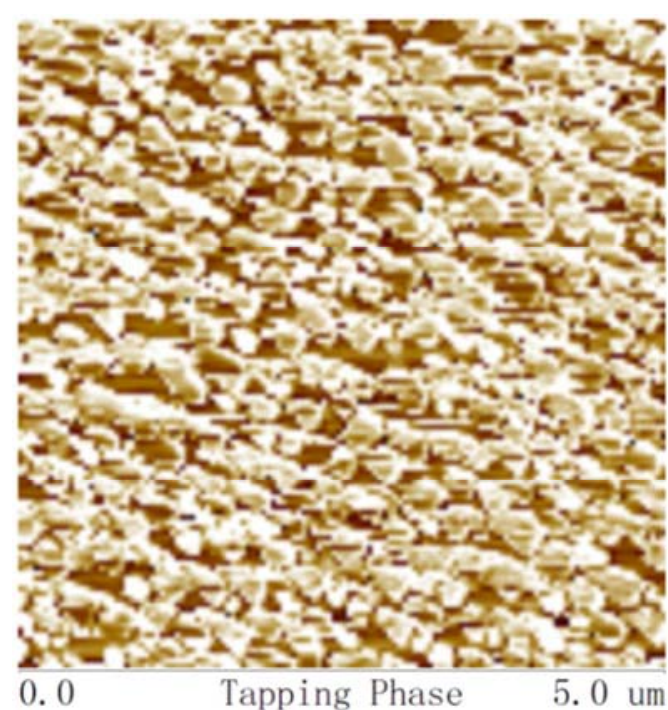

(i) phase mode of $\mathrm{HFBMA}_{12} \mathrm{DMS}_{88} \mathrm{HFBMA}_{12}$

Figure 8. Surface morphology and AFM images.

\subsection{Composition Analysis of the Surface of PHFBMA-b- PDMS-b-PHFBMA}

Figure 9 showed the composition and diagram of the copolymer coatings determined by XPS. The XPS manifestedthat the broad diagram of the triblock polymerswere composed of four strong peaks and three weak peaks, the position of the peak were about $833 \mathrm{eV}, 687 \mathrm{eV}$, $532 \mathrm{eV}, 285 \mathrm{eV}, 151 \mathrm{eV}, 102 \mathrm{eV}$ and $32 \mathrm{eV}$ which were photo ionized by FKLL, F1s, O1s, C1s, Si2s, Si2p and F2s severally $[14,21-22]$. This meant that the surface of the copolymerswere composed of fluorine, carbon, oxygen and silicon. From XPS C1s, it could be seen that there were $\mathrm{CF}_{3}(292.9 \mathrm{eV}), \quad-\mathrm{CF}_{2}(291.4 \mathrm{eV}), \quad-\mathrm{C}=\mathrm{O}(288.6 \mathrm{eV}), \quad-$ $\mathrm{CHF}(286.8 \mathrm{eV}),-\mathrm{COC}=\mathrm{O}(285.1 \mathrm{eV})$ and $-\mathrm{CH}_{\mathrm{n}}(284.6 \mathrm{eV})$ connected with $\mathrm{C}$ directly. Table 2 listed the composition of the copolymer coatings. The contents of fluoride in the coating were $16.7 \%$ and $25.8 \%$, respectively, which were greater than those in the theoretical value $(12.1 \%$ and $19.2 \%$ ), Therefore, it demonstrated that the fluoride contents in the block polymer of the fluorine silicon block were more likely to migrate to the surface.

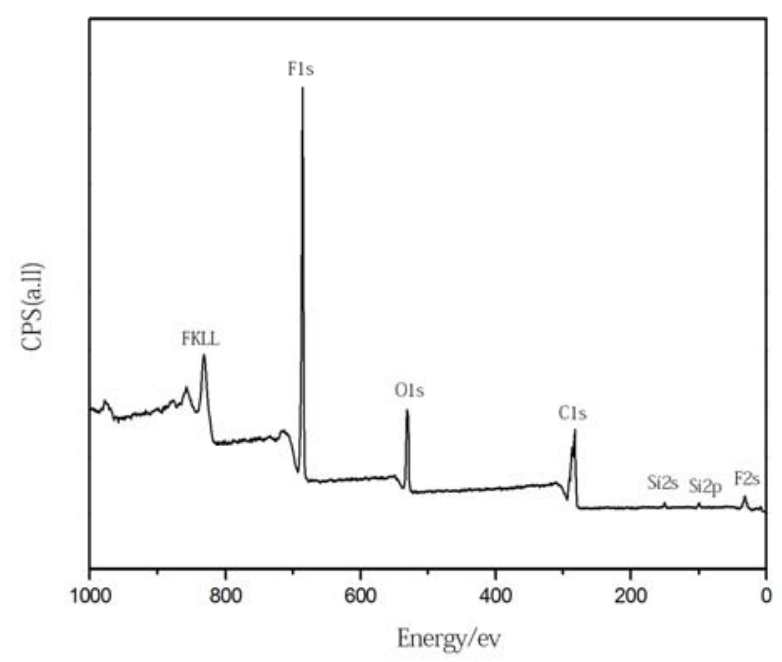

(a) Broad scan of $\mathrm{HFBMA}_{5} \mathrm{DMS}_{88} \mathrm{HFBMA}_{5}$

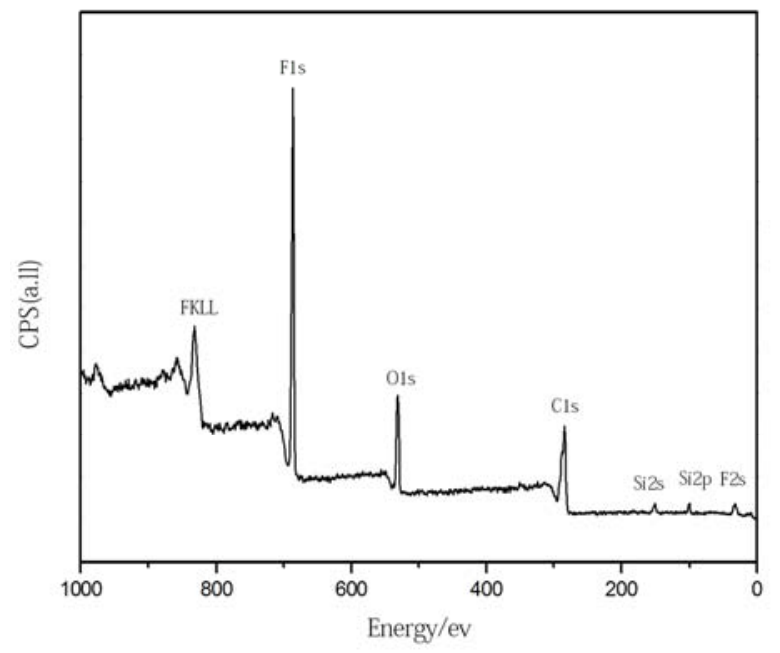

(b) Broad scan of $\mathrm{HFBMA}_{10} \mathrm{DMS}_{88} \mathrm{HFBMA}_{10}$ 


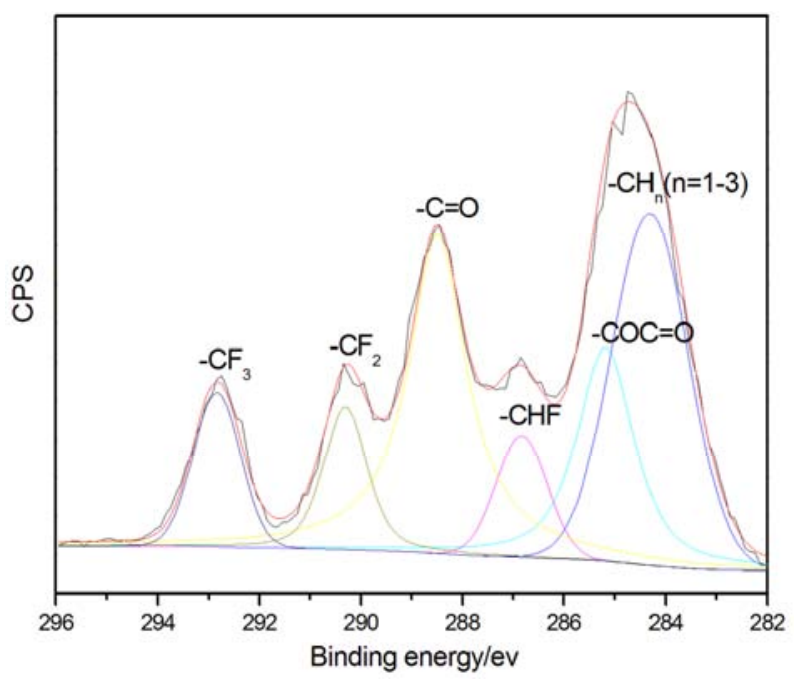

(c) High-resolution $\mathrm{C} 1 \mathrm{~s}$ and fitting spectrumofHFBMA ${ }_{5} \mathrm{DMS}_{88} \mathrm{HFBMA}_{5}$

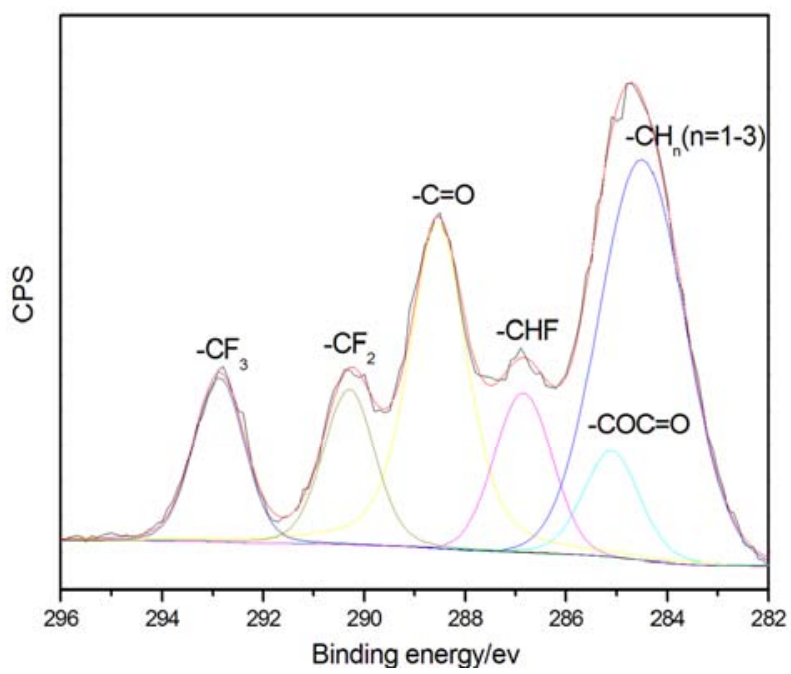

(d) High-resolution $\mathrm{C} 1 \mathrm{~s}$ and fitting spectrumof $\mathrm{HFBMA}_{10} \mathrm{DMS}_{88} \mathrm{HFBMA}_{10}$

Figure 9. XPS of PHFBMA-b-PDMS-b-PHFBMA films.

Table 2. The element content analysis of coating surface.

\begin{tabular}{lllll}
\hline \multirow{2}{*}{ sample } & \multicolumn{4}{l}{ content/\% } \\
\cline { 2 - 5 } & F & C & O & Si \\
\hline HFBMA $_{5}$ DMS $_{88} \mathrm{HFBMA}_{5}$ & 16.7 & 38.3 & 22.3 & 22.7 \\
HFBMA $_{10} \mathrm{DMS}_{88} \mathrm{HFBMA}_{10}$ & 25.8 & 36.7 & 19.4 & 18.1 \\
\hline
\end{tabular}

\section{Conclusion}

PHFBMA-b-PDMS-b-PHFBMA triblock copolymers with designed structures and nanoscopically phase separated surfaces were synthesized perfectly by ATRP. The effects of the fluorine and silicone content on the hydrophobic and oleophobic properties of prepared triblock copolymers were also investigated. The surface energy of the triblock copolymers indirectly obtained from the contact angle reaches as low as $10.43 \mathrm{mN} / \mathrm{m}$ and the fluorine content was only $19.0 \mathrm{wt} \%$. AFM and DSC analysis showed that there was obvious micro phase separation on the prepared copolymers coating. And it could be illustrated from XPS analysis that the fluoride contents in the block polymer of the fluorine silicon block were more likely to migrate to the surface. We would believe that this kind of copolymers has some potential application values especially in self-cleaning coating even heavy-duty materials.

\section{Acknowledgements}

The support of this research by the Key Project of Technology Department of Shaanxi Province of China (No.2012K08-13) is gratefully acknowledged.

\section{References}

[1] Rosati, D., Van Loon, B., and Navard, P. (2000). Rheology of strongly segregated poly (styrene-dimethylsiloxane) block copolymers. Polymer, 41 (1), 367-375.

[2] Liu, Y., Zhu, D., Sun, J., Li, J., Wu, Y., and Gao, C. (2019). Synthesis and characterization of a novel fluorosilicone resin based on trifluoropropylalkoxylsilane. Materials Chemistry and Physics, 224, 40-46.

[3] Zhou, Y., Liu, C., Gao, J., Chen, Y., Yu, F., Chen, M., and Zhang, H. (2019). A novel hydrophobic coating film of waterborne fluoro-silicon polyacrylate polyurethane with properties governed by surface self-segregation. Progress in Organic Coatings, 134, 134-144.

[4] Wang, J., Kong Q., Zhang L., and Qian H. (2018). Preparation and properties of fluorinated low surface energy modified super amphiphobic coatings. Surface Technology, 47 (11), 6672.

[5] Zhu, C., Yang, H., Liang, H., Wang, Z., Dong, J., Xiong, L., and $\mathrm{Xi}$, W. (2018). A novel synthetic UV-curable fluorinated siloxane resin for low surface energy coating. Polymers, 10 (9), 979

[6] Zheng, B., Jiang, G., Wang, W., and Mei, X. (2016). Fabrication of superhydrophilic or superhydrophobic selfcleaning metal surfaces using picosecond laser pulses and chemical fluorination. Radiation Effects and Defects in Solids, $171(5-6), 461-473$.

[7] Yahyaei, H., \& Mohseni, M. (2018). Organic-Inorganic hybrid materials with POSS for coatings. In Polymer/POSS Nanocomposites and Hybrid Materials, pp.395-413. Springer, Cham.

[8] Cao, L., Price, T. P., Weiss, M., and Gao, D. (2008). Super water-and oil-repellent surfaces on intrinsically hydrophilic and oleophilic porous silicon films. Langmuir, 24 (5), 16401643 .

[9] Guo, D., Hou, K., Xu, S., Lin, Y., Li, L., Wen, X., and Pi, P. (2018). Superhydrophobic-superoleophilic stainless steel meshes by spray-coating of a POSS hybrid acrylic polymer for oil-water separation. Journal of materials science, 53 (9), 6403-6413.

[10] Roy, R. E., Indulekha, K., Vijayalakshmi, K. P., Bhuvaneswari, S., Soumyamol, P. B., and Rajeev, R. S. (2019). Fluorosilicone polymers with tailored mechanical, acid resistant and adhesive properties: Role of ultrasonication and functionally active single walled carbon nanotubes. Materials Chemistry and Physics, 223, 523-534. 
[11] Wang, X., Li, X., Lei, Q., Wu, Y., and Li, W. (2018). Fabrication of superhydrophobic composite coating based on fluorosilicone resin and silica nanoparticles. Royal Society open science, 5 (7), 180598.

[12] Huang, L. B., Zhan, X. L., Yi, L. M., Du, F., and Chen, F. Q. (2006). Contact angle and surface tension study of the blends of PS-b-PMTFPS and polystyrene. Polymer Materials Science and Engineering, 22 (2), 141.

[13] Uyanik, N., Yalçinkaya, H., \& Kizilcan, N. (2001). Poly (dimethyl siloxane) -containing five-block copolymers: effects of resin blocks. Surface Coatings International Part B: Coatings Transactions, 84 (4), 309-316.

[14] Wen, X. F., Xie, Q. H., Lu, Y. L., Cai, Z. Q., Pi, P. H., Cheng, J., and Yang, Z. R. (2012). Synthesis of crosslinked fluorinecontaining diblock copolymers by ATRP and its applications on forming the hydrophobic films. Journal of Chemical Engineering of Chinese Universities, 26 (3), 505-510..

[15] Owens, D. K., and Wendt, R. C. (1969). Estimation of the surface free energy of polymers. Journal of applied polymer science, 13 (8), 1741-1747.

[16] Kaelble, D. H. (1970). Dispersion-polar surface tension properties of organic solids. The Journal of Adhesion, 2 (2), 66-81.
[17] Fang, H., Zhou, S., \& Wu, L. (2006). Microphase separation behavior on the surfaces of PEG-MDI-PDMS multiblock copolymer coatings. Applied surface science, 253 (5), 29782983.

[18] Maxson, M. T., Norris, A. W., Owen, M. J., "In Modern Fluoropolymer", J. Scheirs, Ed. John Wiley \& Sons, New York, 1997, pp. 359.

[19] Al-Hussein, M., Serero, Y., Konovalov, O., Mourran, A., Möller, M., and De Jeu, W. H. (2005). Nanoordering of fluorinated side-chain liquid crystalline/amorphous diblock copolymers. Macromolecules, 38 (23), 9610-9616.

[20] Van Rensselar, J. (2017). Silicone and dry-film lubricants. Tribology \& Lubrication Technology, 73 (9), 30.

[21] Kong, J., Wang, M., Zou, J., and An, L. (2015). Soluble and meltable hyperbranched polyborosilazanes toward hightemperature stable SiBCN ceramics. ACS applied materials \& interfaces, 7 (12), 6733-6744.

[22] Zhao, W., Tang, Y., Xi, J., \& Kong, J. (2015). Functionalized graphene sheets with poly (ionic liquid) $\mathrm{s}$ and high adsorption capacity of anionic dyes. Applied Surface Science, 326, 276284. 